\title{
Elliptic Systems of $p$-Laplacian Type
}

\author{
Farah Balaadich and Elhoussine Azroul
}

Abstract. We prove an existence result for solutions of nonlinear $p$-Laplacian systems with data in generalized form:

$$
\left\{\begin{aligned}
-\operatorname{div} \Phi(D u-\Theta(u)) & =f(x, u, D u) \quad \text { in } \Omega \\
u & =0 \text { on } \partial \Omega
\end{aligned}\right.
$$

by the theory of Young measures.

\section{Introduction}

Let $\Omega$ be an open bounded subset of $\mathbb{R}^{n}, n \geq 2$. By $\mathbb{M}^{m \times n}$ we denote the space of $m \times n$ matrices with reduced $\mathbb{R}^{m n}$ topology, equipped with the inner product $A: B=\sum_{i, j} A_{i j} B_{i j}$. If $A \in \mathbb{M}^{m \times n}$, then $|A|$ is the norm of $A$ when regarded as a vector of $\mathbb{R}^{m n}$. Consider the following Dirichlet problem:

$$
\left\{\begin{aligned}
-\operatorname{div} \Phi(D u-\Theta(u)) & =f(x, u, D u) \quad \text { in } \Omega \\
u & =0 \text { on } \partial \Omega,
\end{aligned}\right.
$$

where

$$
\Phi(A)=|A|^{p-2} A, \quad \forall A \in \mathbb{M}^{m \times n},
$$

$\Theta: \mathbb{R}^{m} \rightarrow \mathbb{M}^{m \times n}$ and $f: \Omega \times \mathbb{R}^{m} \times \mathbb{M}^{m \times n} \rightarrow \mathbb{R}^{m}$ are two functions assumed to satisfy some conditions (see below). We give only growth condition on $f($.$) and we point out the importance$ of the Young measure for checking the weak limits of our operator and $f$.

In view of [10], our system (1.1) is a nonlinear degenerate and singular elliptic system according to the cases $p>2$ and $1<p<2$. Throughout this paper, $p$ is a real number such that $p \in(1, \infty)$.

For several decades, there have been intensive research activities for equations, or systems, of $p$-Laplacian types. For example, in [13], we find several types of degenerate elliptic equations 2010 Mathematics Subject Classification. 35J05, 35D30, 28Axx. Key words and phrases. $p$-Laplacian system, Galerkin method, Young measure. Corresponding author: Farah Balaadich. 
for which the existence of a weak solution is discussed by various methods. The authors in [7] proved the existence of a local weak solution and some estimates of $D u$ in $\left[B M O_{l o c}(\Omega)\right]^{N m}$ for the following nonlinear elliptic system $\operatorname{div}\left(|D u|^{p-2} D u\right)=\operatorname{div}\left(|F|^{p-2} F\right)$, for $F \in L_{l o c}^{p}\left(\Omega ; \mathbb{R}^{m}\right)$. Regularity result is achieved in [14] for the second order derivatives of the solution for nonlinear $N$-systems of the $p$-Laplacian type in $n$ space variables. See also [15] for generalized $p$-Laplacian operator with Neumann boundary value problems. F. Crispo et al. [6] proved existence and uniqueness of solutions that are high regular for the $p$-Laplacian system $-\nabla \cdot\left(|\nabla u|^{p-2} \nabla u\right)=$ $f, p \in(1,2)$. Pucci and Servadei [12] established regularity and qualitative properties of the solutions for $p$-Laplacian equations with weights.

When $f$ in (1.1) is independent of $u$ and $D u$, Azroul and Balaadich proved in [2], the existence of weak solutions by using the tool of Young measure. Motivated by the previous work, our aim in this paper is to prove the needed result when $f$ is dependent on $u$ and $D u$. For the utilization of the theory of Young measure in elliptic partial differential equations, we cite for example $[3,4,9]$.

Our assumptions are the following:

(H1) $\Theta: \mathbb{R}^{m} \rightarrow \mathbb{M}^{m \times n}$ is a continuous function satisfying

$$
\Theta(0)=0 \quad \text { and } \quad|\Theta(\xi)-\Theta(\eta)| \leq c|\xi-\eta|
$$

for all $\xi, \eta \in \mathbb{R}^{m}$ and $c$ is a positive constant such that

$$
c<\frac{1}{\operatorname{diam}(\Omega)}\left(\frac{1}{2}\right)^{\frac{1}{p}},
$$

where $\operatorname{diam}(\Omega)$ is the diameter of $\Omega$.

(H2) $f: \Omega \times \mathbb{R}^{m} \times \mathbb{M}^{m \times n} \rightarrow \mathbb{R}^{m}$ is a Carathéodory function (i.e. $x \mapsto f(x, u, A$ ) is measurable for every $(u, A) \in \mathbb{R}^{m} \times \mathbb{M}^{m \times n}$ and $(u, A) \mapsto f(x, u, A)$ is continuous for almost every $\left.x \in \Omega\right)$. Moreover, we assume that the following two additional conditions hold:

(i) For constants $0<\gamma<p-1,0 \leq \mu<p-1$ and a function $d \in L^{p^{\prime}}(\Omega)$ there holds

$$
|f(x, \xi, A)| \leq d(x)+|\xi|^{\gamma}+|A|^{\mu}
$$

for a.e. $x \in \Omega$ and all $(\xi, A) \in \mathbb{R}^{m} \times \mathbb{M}^{m \times n}$.

(ii) In addition to (i), the function $f$ is independent of the third variable, or, for almost $x \in \Omega$ and all $u \in \mathbb{R}^{m}$, the mapping $A \mapsto f(x, u, A)$ is linear.

Remark 1. Note that, the constants $\gamma, \mu$ in (H2)(i) and $c$ in (H1) allow to obtain the coercivity of the mapping $T$ defined in section 3 . 
It is our purpose in this paper to prove an existence theorem for problems corresponding to (1.1) when $f$ is of general form. So that, we generalize our work [2].

We now introduce the definition of a weak solution for (1.1) as follows:

Definition 1. We say that $u \in W_{0}^{1, p}\left(\Omega ; \mathbb{R}^{m}\right)$ is a weak solution to (1.1) if

$$
\int_{\Omega} \Phi(D u-\Theta(u)): D \varphi d x=\int_{\Omega} f(x, u, D u) \cdot \varphi d x
$$

holds for all $\varphi \in W_{0}^{1, p}\left(\Omega ; \mathbb{R}^{m}\right)$.

We shall prove the following existence theorem.

Theorem 1.1. Assume that $\Theta$ satisfies the condition (H1) for some $p \in(1, \infty)$. Then for every $f$ satisfying (H2), the problem (1.1) has a weak solution in the sense of Definition 1.

\section{Preliminaries and useful properties}

Let $\Omega$ be a bounded open subset of $\mathbb{R}^{n}, n \geq 2$, with smooth boundary $\partial \Omega$. In the sequel, the Hölder inequality and the following Poincaré inequality (see [11, Lemma 2.2]); there exists a positive constant $\alpha=\operatorname{diam}(\Omega)$ such that

$$
\|z\|_{p} \leq \frac{\alpha}{2}\|D z\|_{p}, \quad \forall z \in W_{0}^{1, p}\left(\Omega ; \mathbb{R}^{m}\right),
$$

are central to establish the required estimates to prove the desired results. As mentioned before, we will use the tool of Young measure to prove our Theorem 1.1. In the following, we briefly summarize some useful properties needed in the sequel.

By $C_{0}\left(\mathbb{R}^{m}\right)$ we denote the closure of the space of continuous functions on $\mathbb{R}^{m}$ with compact support with respect to the $\|\cdot\|_{\infty}$-norm. Its dual can be identified with $\mathcal{M}\left(\mathbb{R}^{m}\right)$, the space of signed Radon measures with finite mass. The related duality pairing is given by

$$
\langle\nu, g\rangle=\int_{\mathbb{R}^{m}} g(\lambda) d \nu(\lambda)
$$

for $\nu: \Omega \rightarrow \mathcal{M}\left(\mathbb{R}^{m}\right)$ and $g \in C_{0}\left(\mathbb{R}^{m}\right)$.

Lemma 2.1. ([8, Theorem 1.5.2]) Let $\left\{z_{j}\right\}_{j \geq 1}$ be a bounded sequence in $L^{\infty}\left(\Omega ; \mathbb{R}^{m}\right)$. Then there exist a subsequence $\left\{z_{k}\right\}_{k \geq 1} \subset\left\{z_{j}\right\}_{j \geq 1}$ and a Borel probability measure $\nu_{x}$ on $\mathbb{R}^{m}$ for a.e. $x \in \Omega$, such that for almost each $g \in C\left(\mathbb{R}^{m}\right)$ we have

$$
g\left(z_{k}\right) \rightarrow^{*} \bar{g} \text { weaklyin } L^{\infty}\left(\Omega ; \mathbb{R}^{m}\right),
$$

where $\bar{g}(x)=\left\langle\nu_{x}, g\right\rangle=\int_{\mathbb{R}^{m}} g(\lambda) d \nu_{x}(\lambda)$ for a.e. $x \in \Omega$. 
Note that, when $z_{k}=D v_{k}$ for $v_{k}: \Omega \rightarrow \mathbb{R}^{m}$, then the above integrand remains true on $\mathbb{M}^{m \times n}$, i.e.

$$
\left\langle\nu_{x}, g\right\rangle=\int_{\mathbb{M}^{m \times n}} g(\lambda) d \nu_{x}(\lambda),
$$

and we call $\left\{\nu_{x}\right\}_{x \in \Omega}$ the family of Young measures associated with $\left\{D v_{k}\right\}_{k}$. In [5], it is shown that for any Carathéodory function $g: \Omega \times \mathbb{R}^{m} \rightarrow \mathbb{R}$ and $\left(z_{k}\right)_{k}$ generates a Young measure $\nu_{x}$, we have

$$
g\left(x, z_{k}\right) \rightarrow\left\langle\nu_{x}, g(x, .)\right\rangle=\int_{\mathbb{R}^{m}} g(x, \lambda) d \nu_{x}(\lambda)
$$

weakly in $L^{1}\left(\Omega^{\prime}\right)$ for all measurable $\Omega^{\prime} \subset \Omega$, provided that the negative part $g^{-}\left(x, z_{k}\right)$ is equiintegrable. We end this section by recalling the following useful lemma:

Lemma 2.2. ([1]) Let $\xi, \eta \in \mathbb{R}^{m}$ and let $p \in(1, \infty)$. We have

$$
|\xi|^{p-2} \xi \cdot(\xi-\eta) \geq \frac{1}{p}|\xi|^{p}-\frac{1}{p}|\eta|^{p} .
$$

\section{Proof of the main result}

In this section, we give the proof of Theorem 1.1 basing on the Galerkin method to construct the approximating solutions and the theory of Young measure for the passage to the limit. Let consider the operator $T: W_{0}^{1, p}\left(\Omega ; \mathbb{R}^{m}\right) \rightarrow W^{-1, p^{\prime}}\left(\Omega ; \mathbb{R}^{m}\right)$ defined by

$$
\langle T(u), \varphi\rangle=\int_{\Omega} \Phi(D u-\Theta(u)): D \varphi d x-\int_{\Omega} f(x, u, D u) \cdot \varphi d x .
$$

Remark that our problem (1.1) is equivalent to find $u \in W_{0}^{1, p}\left(\Omega ; \mathbb{R}^{m}\right)$ for which $\langle T(u), \varphi\rangle=0$ for all $\varphi \in W_{0}^{1, p}\left(\Omega ; \mathbb{R}^{m}\right)$. Here $\langle.,$.$\rangle denote the duality pairing of W_{0}^{1, p}\left(\Omega ; \mathbb{R}^{m}\right)$ and its dual. In the sequel, the value of the positive constant $C$ may change from line to line.

To prove the existence of the approximating solutions, we proceed by the following assertions: Assertion 1: $T(u)$ is linear, well defined and bounded.

Indeed, for arbitrary $u \in W_{0}^{1, p}\left(\Omega ; \mathbb{R}^{m}\right), T(u)$ is trivially linear and (without loss of generality, we may assume that $\gamma=p-1=\mu$ )

$$
\begin{aligned}
|\langle T(u), \varphi\rangle| & =\left|\int_{\Omega} \Phi(D u-\Theta(u)): D \varphi d x-\int_{\Omega} f(x, u, D u) \cdot \varphi d x\right| \\
& \leq \int_{\Omega}|D u-\Theta(u)|^{p-1}|D \varphi| d x+\int_{\Omega}|f(x, u, D u) \| \varphi| d x \\
& \leq\left(\int_{\Omega}|D u-\Theta(u)|^{p} d x\right)^{\frac{1}{p^{\prime}}}\|D \varphi\|_{p}+\left(\|d\|_{p^{\prime}}+\|u\|_{p}^{p-1}+\|D u\|_{p}^{p-1}\right)\|\varphi\|_{p} \\
& \leq 2^{\frac{(p-1)^{2}}{p}}\left(\int_{\Omega}|D u|^{p}+|\Theta(u)| d x\right)^{\frac{1}{p^{\prime}}}\|D \varphi\|_{p}+\left(\|d\|_{p^{\prime}}+\|u\|_{p}^{p-1}+\|D u\|_{p}^{p-1}\right)\|\varphi\|_{p}
\end{aligned}
$$




$$
\leq C\|\varphi\|_{1, p}
$$

where we have used the Poincaré inequality, the Eq. (1.2) and (2.1) and the following inequality

$$
|a+b|^{p} \leq 2^{p-1}\left(|a|^{p}+|b|^{p}\right) \quad \text { for } \quad p>1 \text {. }
$$

Thus $T$ is well defined and bounded.

Assertion 2: The restriction of $T$ to a finite linear subspace of $W_{0}^{1, p}\left(\Omega ; \mathbb{R}^{m}\right)$ is continuous.

Indeed, let $W$ be a finite subspace of $W_{0}^{1, p}\left(\Omega ; \mathbb{R}^{m}\right)$ with $\operatorname{dim} W=r$ and $\left(w_{i}\right)_{i=1, . ., r}$ a basis of $W$. Let $\left(u_{k}=a_{k}^{i} w_{i}\right)$ be a sequence in $W$ which converges to $u=a^{i} w_{i}$ in $W$ (with conventional summation). Then $u_{k} \rightarrow u$ and $D u_{k} \rightarrow D u$ almost everywhere for a subsequence still denoted by $\left(u_{k}\right)$. On the one hand, the continuity of $\Theta$ and $f$ implies that

$$
\Phi\left(D u_{k}-\Theta\left(u_{k}\right)\right) \rightarrow \Phi(D u-\Theta(u))
$$

and

$$
f\left(x, u_{k}, D u_{k}\right) \rightarrow f(x, u, D u)
$$

almost everywhere. On the other hand, since $u_{k} \rightarrow u$ strongly in $W$

$$
\int_{\Omega}\left|u_{k}-u\right|^{p} d x \rightarrow 0 \text { and } \quad \int_{\Omega}\left|D u_{k}-D u\right|^{p} d x \rightarrow 0 .
$$

Thus, there exists a subsequence of $\left(u_{k}\right)$ still denoted by $\left(u_{k}\right)$ and $g_{1}, g_{2} \in L^{1}(\Omega)$ such that $\left|u_{k}-u\right|^{p} \leq g_{1}$ and $\left|D u_{k}-D u\right|^{p} \leq g_{2}$. According to (3.1), we get

$$
\begin{aligned}
\left|u_{k}\right|^{p}=\left|u_{k}-u+u\right|^{p} & \leq 2^{p-1}\left(\left|u_{k}-u\right|^{p}+|u|^{p}\right) \\
& \leq 2^{p-1}\left(g_{1}+|u|^{p}\right) .
\end{aligned}
$$

Similarly

$$
\left|D u_{k}\right|^{p} \leq 2^{p-1}\left(g_{2}+|D u|^{p}\right) .
$$

Consequently, $\left\|u_{k}\right\|_{p}$ and $\left\|D u_{k}\right\|_{p}$ are bounded by a constant $C$. Now, in order to apply the Vitali Theorem, we show that the sequences $\left(\Phi\left(D u_{k}-\Theta\left(u_{k}\right)\right): D \varphi\right)$ and $\left(f\left(x, u_{k}, D u_{k}\right) \cdot \varphi\right)$ are equiintegrable. To do this, we take $\Omega^{\prime} \subset \Omega$ to be measurable, then by the assertion 1 , we have (without loss of generality, we may assume that $\gamma=p-1=\mu$ )

$$
\int_{\Omega^{\prime}}\left|\Phi\left(D u_{k}-\Theta\left(u_{k}\right)\right): D \varphi\right| d x \leq 2^{\frac{(p-1)^{2}}{p}}(\underbrace{\left\|D u_{k}\right\|_{p}^{p}}_{\leq C}+c^{p} \underbrace{\left\|u_{k}\right\|_{p}^{p}}_{\leq C})^{\frac{1}{p^{\prime}}}\left(\int_{\Omega^{\prime}}|D \varphi|^{p} d x\right)^{\frac{1}{p}}
$$

and

$$
\int_{\Omega^{\prime}}\left|f\left(x, u_{k}, D u_{k}\right) \cdot \varphi\right| d x \leq \frac{\alpha}{2}(\|d\|_{p^{\prime}}+\underbrace{\left\|u_{k}\right\|_{p}^{p-1}}_{\leq C}+\underbrace{\left\|D u_{k}\right\|_{p}^{p-1}}_{\leq C})\left(\int_{\Omega^{\prime}}|D \varphi|^{p} d x\right)^{\frac{1}{p}} .
$$


Since $\int_{\Omega^{\prime}}|D \varphi|^{p} d x$ is arbitrary small if the measure of $\Omega^{\prime}$ is chosen small enough, then the equiintegrability of $\left(\Phi\left(D u_{k}-\Theta\left(u_{k}\right)\right): D \varphi\right)$ and $\left(f\left(x, u_{k}, D u_{k}\right) \cdot \varphi\right)$ follows. By virtue of the Vitali Theorem, $T$ is continuous.

Assertion 3: $T$ is coercive.

Indeed, by taking $\varphi=u$ in the definition of $T$, we have

$$
\langle T(u), u\rangle=\int_{\Omega} \Phi(D u-\Theta(u)): D u d x-\int_{\Omega} f(x, u, D u) \cdot u d x .
$$

To prove the coercivity of $T$ we argue as follows:

By Lemma 2.2, we have

$$
|A|^{p-2} A:(A-B) \geq \frac{1}{p}|A|^{p}-\frac{1}{p}|B|^{p},
$$

then by taking $A=D u-\Theta(u)$ and $B=-\Theta(u)$, we obtain

$$
\begin{aligned}
|D u-\Theta(u)|^{p-2} & (D u-\Theta(u)): D u \\
& =|D u-\Theta(u)|^{p-2}(D u-\Theta(u)):(D u-\Theta(u)+\Theta(u)) \\
& \geq \frac{1}{p}|D u-\Theta(u)|^{p}-\frac{1}{p}|\Theta(u)|^{p} .
\end{aligned}
$$

Since

$$
\begin{aligned}
\frac{1}{2^{p-1}}|D u|^{p} & =\frac{1}{2^{p-1}}|D u-\Theta(u)+\Theta(u)|^{p} \\
& \leq \frac{1}{2^{p-1}}\left[2^{p-1}\left(|D u-\Theta(u)|^{p}+|\Theta(u)|^{p}\right)\right] \\
& =|D u-\Theta(u)|^{p}+|\Theta(u)|^{p}
\end{aligned}
$$

then (3.3) and (1.2) implies

$$
\begin{aligned}
|D u-\Theta(u)|^{p-2}(D u-\Theta(u)): D u & \geq \frac{1}{p} \frac{1}{2^{p-1}}|D u|^{p}-\frac{2}{p}|\Theta(u)|^{p} \\
& \geq \frac{1}{p} \frac{1}{2^{p-1}}|D u|^{p}-\frac{2}{p} c^{p}|u|^{p} .
\end{aligned}
$$

By the Hölder inequality, the Eq. (2.1) and the condition (H2)(i), we get

$$
\begin{aligned}
\left|\int_{\Omega} f(x, u, D u) \cdot u d x\right| & \leq \int_{\Omega} d(x)|u| d x+\int_{\Omega}|u|^{\gamma}|u| d x+\int_{\Omega}|D u|^{\mu}|u| d x \\
& \leq\|d\|_{p^{\prime}}\|u\|_{p}+\|u\|_{\gamma p^{\prime}}^{\gamma}\|u\|_{p}+\|D u\|_{\mu p^{\prime}}^{\mu}\|u\|_{p} \\
& \leq \frac{\alpha}{2}\|d\|_{p^{\prime}}\|D u\|_{p}+\left(\frac{\alpha}{2}\right)^{\gamma+1}\|D u\|_{p}^{\gamma+1}+\frac{\alpha}{2}\|D u\|_{p}^{\mu+1} .
\end{aligned}
$$


Consequently, owing to (3.2), by (2.1) and the choice of the constant $c$ in (H1), we obtain

$$
\begin{aligned}
& \langle T(u), u\rangle \\
& \geq \frac{1}{p} \frac{1}{2^{p-1}} \int_{\Omega}|D u|^{p} d x-\frac{2}{p} c^{p} \int_{\Omega}|u|^{p} d x-\frac{\alpha}{2}\|d\|_{p^{\prime}}\|D u\|_{p}-\left(\frac{\alpha}{2}\right)^{\gamma+1}\|D u\|_{p}^{\gamma+1}-\frac{\alpha}{2}\|D u\|_{p}^{\mu+1} \\
& \geq \frac{1}{p} \frac{1}{2^{p}} \int_{\Omega}|D u|^{p} d x-\frac{\alpha}{2}\|d\|_{p^{\prime}}\|D u\|_{p}-\left(\frac{\alpha}{2}\right)^{\gamma+1}\|D u\|_{p}^{\gamma+1}-\frac{\alpha}{2}\|D u\|_{p}^{\mu+1} .
\end{aligned}
$$

From the above estimation it follows that

$$
\langle T(u), u\rangle \rightarrow \infty \quad \text { as } \quad\|u\|_{1, p} \rightarrow \infty
$$

since $p>\max \{1, \gamma+1, \mu+1\}$.

Now, we have all ingredients to construct the approximating solutions by the Galerkin method. Let $W_{1} \subset W_{2} \subset . . \subset W_{0}^{1, p}\left(\Omega ; \mathbb{R}^{m}\right)$ be a sequence of finite dimensional subspaces with the property that $\cup_{k \geq 1} W_{k}$ is dense in $W_{0}^{1, p}\left(\Omega ; \mathbb{R}^{m}\right)$. Such a sequence $\left(W_{k}\right)$ exists since $W_{0}^{1, p}\left(\Omega ; \mathbb{R}^{m}\right)$ is separable. Let fix $k$ and assume that $\operatorname{dim} W_{k}=r$. For simplicity, we write $\sum_{1 \leq i \leq r} a^{i} w_{i}=a^{i} w_{i}$ where $\left(w_{i}\right)_{i=1}^{r}$ is a basis of $W_{k}$. Define the mapping

$$
\begin{aligned}
G: \mathbb{R}^{r} & \longrightarrow \mathbb{R}^{r} \\
\left(a^{1}, \ldots, a^{r}\right) & \rightarrow\left(\left\langle T\left(a^{i} w_{i}\right), w_{j}\right\rangle\right)_{j=1, . ., r}
\end{aligned}
$$

Lemma 3.1. $G$ is continuous and $G(a) . a \rightarrow \infty$ as $\|a\|_{\mathbb{R}^{r}} \rightarrow \infty$.

Proof. Since $T$ restricted to $W_{k}$ is continuous by the assertion 2, $G$ is continuous. Let be $a \in \mathbb{R}^{r}$ and $u=a^{i} w_{i} \in W_{k}$. Then $\|a\|_{\mathbb{R}^{r}} \rightarrow \infty$ is equivalent to $\|u\|_{1, p} \rightarrow \infty$ and

$$
G(a) \cdot a=\langle T(u), u\rangle .
$$

Assertion 3 gives $G(a) . a \rightarrow \infty$ when $\|a\|_{\mathbb{R}^{r}} \rightarrow \infty$.

Lemma 3.2. For all $k \in \mathbb{N}$ there exists $u_{k} \in W_{k}$ such that

$$
\left\langle T\left(u_{k}\right), \varphi\right\rangle=0 \text { for all } \varphi \in W_{k},
$$

and there is a constant $R>0$ such that

$$
\left\|u_{k}\right\|_{1, p} \leq R \quad \text { for all } \quad k \in \mathbb{N} \text {. }
$$

Proof. Since $G(a) . a \rightarrow \infty$ as $\|a\|_{\mathbb{R}^{r}} \rightarrow \infty$ by Lemma 3.1, it follows the existence of $R>0$ such that for all $a \in \partial B_{R}(0) \subset \mathbb{R}^{r}$ we have $G(a) . a>0$. Thanks to the usual topological arguments (see e.g. [16, Proposition 2.8]), $G(x)=0$ has a solution $x \in B_{R}(0)$. Hence, for all 
$k \in \mathbb{N}$ there exists $u_{k} \in W_{k}$ such that (3.4) holds. On the other hand, since $\langle T(u), u\rangle \rightarrow \infty$ as $\|u\|_{1, p} \rightarrow \infty$, it follows that there exists $R>0$ with the property, that $\langle T(u), u\rangle>1$ whenever $\|u\|_{1, p}>R$. Consequently, for the sequence of Galerkin approximations $u_{k} \in W_{k}$ which satisfy $\left\langle T\left(u_{k}\right), u_{k}\right\rangle=0$ by (3.4), it follows the uniform bound (3.5).

Proof of Theorem 1.1. Let $\left\{u_{k}\right\}$ be the sequence defined in Lemma 3.2. According to Lemma 2.1 and the Eq. (3.5), there exists a Young measure $\nu_{x}$ generated by $D u_{k}$. Now, thanks to [2, Lemma 4.1], we recover some facts about the Young measure $\nu=\left\{\nu_{x}\right\}_{x \in \Omega}$ as follows:

(a) $\nu_{x}$ is a probability measure, i.e. $\left\|\nu_{x}\right\|_{\mathcal{M}\left(\mathbb{M}^{m \times n}\right)}=1$ for a.e. $x \in \Omega$.

(b) The weak $L^{1}$-limit of $D u_{k}$ is given by

$$
\left\langle\nu_{x}, i d\right\rangle=\int_{\mathbb{M}^{m \times n}} \lambda d \nu_{x}(\lambda)
$$

(c) $\nu_{x}$ satisfies $\left\langle\nu_{x}, i d\right\rangle=D u(x)$ for a.e. $x \in \Omega$.

The proof of Theorem 1.1 is divided into two cases, accordingly to the assumptions (i) and (ii): Case (i): Let $E_{k, \epsilon}=\left\{x \in \Omega:\left|u_{k}(x)-u(x)\right| \geq \epsilon\right\}$ for $\epsilon$ positive. By (3.5), we have (for a subsequence) $u_{k} \rightarrow u$ in $L^{p}\left(\Omega ; \mathbb{R}^{m}\right)$. Since

$$
\int_{\Omega}\left|u_{k}-u\right|^{p} d x \geq \int_{E_{k, \epsilon}}\left|u_{k}-u\right|^{p} d x \geq \epsilon^{p}\left|E_{k, \epsilon}\right|,
$$

it follows that

$$
\left|E_{k, \epsilon}\right| \leq \frac{1}{\epsilon^{p}} \int_{\Omega}\left|u_{k}-u\right|^{p} d x \rightarrow 0 \quad \text { as } \quad k \rightarrow \infty .
$$

Hence $u_{k} \rightarrow u$ in measure for $k \rightarrow \infty$, and we may infer that, after extraction of a suitable subsequence, if necessary

$$
u_{k} \rightarrow u \quad \text { almost everywhere for } \quad k \rightarrow \infty \text {. }
$$

The continuity of the function $\Theta$ and the weak limit defined in the above properties (a) and (b) implies that

$$
\begin{aligned}
D u_{k}-\Theta\left(u_{k}\right) \rightarrow & \int_{\mathbb{M}^{m \times n}}(\lambda-\Theta(u)) d \nu_{x}(\lambda) \\
& =\underbrace{\int_{\mathbb{M}^{m} m n} \lambda d \nu_{x}(\lambda)}_{=: D u(x)}-\Theta(u) \underbrace{\int_{\mathbb{M}^{m \times}} d \nu_{x}(\lambda)}_{=: 1} \\
& =D u-\Theta(u)
\end{aligned}
$$


weakly in $L^{1}(\Omega)$, since $\left(D u_{k}-\Theta\left(u_{k}\right)\right)$ is equiintegrable by (1.2). Therefore

$$
\Phi\left(D u_{k}-\Theta\left(u_{k}\right)\right) \rightarrow \Phi(D u-\Theta(u))
$$

weakly in $L^{1}(\Omega)$. Since $L^{p}(\Omega)$ is reflexive and $\Phi\left(D u_{k}-\Theta\left(u_{k}\right)\right)$ is bounded (see Assertion 1 if necessary), the sequence $\left\{\Phi\left(D u_{k}-\Theta\left(u_{k}\right)\right)\right\}$ converges in $L^{p^{\prime}}(\Omega)$. Hence its weak $L^{p^{\prime}}$-limit is also $\Phi(D u-\Theta(u))$. We may infer that

$$
\lim _{k \rightarrow \infty} \int_{\Omega} \Phi\left(D u_{k}-\Theta\left(u_{k}\right)\right): D \varphi d x=\int_{\Omega} \Phi(D u-\Theta(u)): D \varphi d x \quad \forall \varphi \in \cup_{k \geq 1} W_{k} .
$$

To pass to the limit on the source term, we need the convergence almost everywhere of $D u_{k}$. Similarly to $E_{k, \epsilon}$, we consider $F_{k, \epsilon}=\left\{x \in \Omega:\left|D u_{k}(x)-D u(x)\right| \geq \epsilon\right\}$, then

$$
D u_{k} \rightarrow D u \quad \text { in measure for } \quad k \rightarrow \infty
$$

Thus (for a subsequence), $D u_{k} \rightarrow D u$ almost everywhere for $k \rightarrow \infty$. The continuity of $f$ permit to deduce that

$$
f\left(x, u_{k}, D u_{k}\right) \cdot \varphi \rightarrow f(x, u, D u) \cdot \varphi
$$

for arbitrary $\varphi \in W_{0}^{1, p}\left(\Omega ; \mathbb{R}^{m}\right)$. By the growth condition (H2)(i), we have $\left(f\left(x, u_{k}, D u_{k}\right) \cdot \varphi(x)\right)$ is equiintegrable (see Assertion 2 if necessary), thus $f\left(x, u_{k}, D u_{k}\right) \cdot \varphi(x) \rightarrow f(x, u, D u) \cdot \varphi(x)$ in $L^{1}(\Omega)$ by the Vitali Convergence Theorem. This implies

$$
\lim _{k \rightarrow \infty} \int_{\Omega} f\left(x, u_{k}, D u_{k}\right) \cdot \varphi(x) d x=\int_{\Omega} f(x, u, D u) \cdot \varphi(x) d x, \quad \forall \varphi \underset{k \geq 1}{\cup} W_{k} .
$$

Case (ii): If $f$ is independent of the third variable, we easily verify that

$$
f\left(x, u_{k}\right) \rightarrow f(x, u) \text { in } L^{p^{\prime}}(\Omega) .
$$

In the other situation, we have that, for a.e. $x \in \Omega$ and all $u \in \mathbb{R}^{m}$, the mapping $A \mapsto f(x, u, A)$ is linear. Here we argue as follows to identify the weak limit of $f\left(x, u_{k}, D u_{k}\right)$ :

$$
\begin{aligned}
f\left(x, u_{k}, D u_{k}\right) \rightarrow\left\langle\nu_{x}, f(x, u, .)\right\rangle & =\int_{\mathbb{M}^{m \times n}} f(x, u, \lambda) d \nu_{x}(\lambda) \\
& =f(x, u, .) o \underbrace{\int_{\mathbb{M}^{m \times n}} \lambda d \nu_{x}(\lambda)}_{=: D u(x)} \\
& =f(x, u, D u),
\end{aligned}
$$

since $f\left(x, u_{k}, D u_{k}\right)$ is equiintegrable. 


\section{Conclusion}

For every $\varphi \in W_{0}^{1, p}\left(\Omega ; \mathbb{R}^{m}\right)$, since $\underset{k \geq 1}{\cup} W_{k}$ is dense in $W_{0}^{1, p}\left(\Omega ; \mathbb{R}^{m}\right)$, there exists a sequence $\left\{\varphi_{k}\right\} \subset \underset{k \geq 1}{\cup} W_{k}$ such that $\varphi_{k} \rightarrow \varphi$ in $W_{0}^{1, p}\left(\Omega ; \mathbb{R}^{m}\right)$ as $k \rightarrow \infty$. We can now pass to the limit in the Galerkin equations:

$$
\begin{aligned}
& \left\langle T\left(u_{k}\right), \varphi_{k}\right\rangle-\langle T(u), \varphi\rangle \\
& =\int_{\Omega} \Phi\left(D u_{k}-\Theta\left(u_{k}\right)\right): D \varphi_{k} d x-\int_{\Omega} \Phi(D u-\Theta(u)): D \varphi d x \\
& -\int_{\Omega} f\left(x, u_{k}, D u_{k}\right) \cdot \varphi_{k} d x+\int_{\Omega} f(x, u, D u) \cdot \varphi d x \\
& =\int_{\Omega} \Phi\left(D u_{k}-\Theta\left(u_{k}\right)\right):\left(D \varphi_{k}-D \varphi\right) d x+\int_{\Omega}\left(\Phi\left(D u_{k}-\Theta\left(u_{k}\right)\right)-\Phi(D u-\Theta(u))\right): D \varphi d x \\
& -\int_{\Omega} f\left(x, u_{k}, D u_{k}\right) \cdot\left(\varphi_{k}-\varphi\right) d x-\int_{\Omega}\left(f\left(x, u_{k}, D u_{k}\right)-f(x, u, D u)\right) \cdot \varphi d x .
\end{aligned}
$$

The right hand side of the above equation tends to zero as $k$ tends to infinity by the previous results. By virtue of Lemma 3.2, it follows that $\langle T(u), \varphi\rangle=0$ for all $\varphi \in W_{0}^{1, p}\left(\Omega ; \mathbb{R}^{m}\right)$ as desired.

\section{References}

[1] A. Abassi, A. El Hachimi and A. Jamea, Entropy solutions to nonlinear Neumann problems with $L^{1}$-data, Int. J. Math. Statist. 2 (2008), 4-17.

[2] E. Azroul and F. Balaadich, Weak solutions for generalized $p$-Laplacian systems via Young measures, Moroccan J. of Pure and Appl. Anal. (MJPAA), 4 (2) (2018, 77-84.

[3] E. Azroul and F. Balaadich, Quasilinear elliptic systems in perturbed form, Int. J. Nonlinear Anal. Appl. 10 (2019) No. 2, 255-266.

[4] E. Azroul and F. Balaadich, A weak solution to quasilinear elliptic problems with perturbed gradient, Rend. Circ. Mat. Palermo, II. Ser 70, 151-166 (2021). https://doi.org/10.1007/s12215-020-00488-4

[5] J. M. Ball, A version of the fundamental theorem for Young measures. In: PDEs and Continuum Models of Phase Transitions (Nice, 1988). Lecture Notes in Phys, 344 (1989), 207-215.

[6] F. Crispo, C-R. Grisanti and P. Maremont, On the high regularity of solutions to the $p$-Laplacian boundary value problem in exterior domains, Ann. Math. Pure Appl. 195 (2016), 821-834. 
[7] E. DiBenedetto and J. Manfredi, On the Higher Integrability of the Gradient of Weak Solutions of Certain Degenerate Elliptic Systems, American Journal of Mathematics, 115 (5) (1993), 1107-1134.

[8] LC. Evans, Weak Convergence Methods for Nonlinear Partial Differential Equations, Am. Math. Soc., New York 1990.

[9] N. Hungerbühler, Quasilinear elliptic systems in divergence form with weak monotonicity, New York J. Math. 5 (1999), 83-90.

[10] T. Iwaniec, Projections onto gradients fields and $L^{p}$-estimates for degenrated elliptic operators, Studia Math., 75 (3) (1983), 293-312.

[11] G M. Lieberman, The natural generalizationj of the natural conditions of ladyzhenskaya and uraltseva for elliptic equations, Commun. In. Partial Differential Equations. 16(2\&3) (1991), 311-361.

[12] P. Pucci and R. Servadei, On weak solutions for $p$-Laplacian equations with weights, Rend. Lincei Mat. Appl. 18 (2007), 257-267.

[13] M. Struwe, Variational Methods, Springer Verlag Berlin, Heidelberg, New York, second edition 1996.

[14] H. Beirão da Veiga and F. Crispo, On the global $W^{2, q}$ regularity for nonlinear N-systems of the $p$-Laplacian type in $\mathrm{n}$ space variables, Nonlinear Anal. 75 (2012), 4346-4354.

[15] L. Wei and R P. Agarwal, Existence of solutions to nonlinear Neumann boundary value problems with generalized p-Laplacian operator, Comput. Math. Appl., 56 (2008), 530-541.

[16] E. Zeidler, Nonlinear functional analysis and its application, volume I. Springer, 1986.

Farah Balaadich University of Sidi Mohamed Ben Abdellah, Faculty of Sciences Dhar El Mehraz, B.P. 1796 Atlas, Fez-Morocco

E-mail: balaadich.edp@gmail.com

Elhoussine Azroul University of Sidi Mohamed Ben Abdellah, Faculty of Sciences Dhar El Mehraz, B.P. 1796 Atlas, Fez-Morocco

E-mail: elhoussine.azroul@gmail.com 